This is the peer reviewed version of the following article: Comerford, D. A., and Robinson, J. (2017) Die-by Framing both Lengthens and Shortens Life: Further Evidence on Constructed Beliefs in Life Expectancy. J. Behav. Dec. Making, 30: 1104-1112, which has been published in final form at https://doi.org/10.1002/bdm.2027. This article may be used for non-commercial purposes in accordance With Wiley Terms and Conditions for self-archiving. 


\section{JOURNAL OF \\ BEHAVIORAL DECISION MAKING}

\section{Die-by framing both lengthens and shortens life: Further evidence on constructed beliefs in life expectancy}

\begin{tabular}{|r|l|}
\hline Journal: & Journal of Behavioral Decision Making \\
\hline Manuscript ID & BDM-17-0089.R2 \\
\hline Wiley - Manuscript type: & Research Article \\
\hline Date Submitted by the Author: & 07-Jun-2017 \\
\hline Complete List of Authors: & $\begin{array}{l}\text { comerford, david; university of stirling, economics } \\
\text { Robinson, Jenny; Irrational Insights Ltd., Research Office }\end{array}$ \\
\hline Keywords: & Life Expectancy, Probabilistic Reasoning, Framing Effects, Annuities \\
\hline & \\
\hline
\end{tabular}

SCHOLARONE ${ }^{\text {m }}$

Manuscripts 


\title{
Die-by framing both lengthens and shortens life: Further evidence on constructed beliefs in life expectancy
}

\begin{abstract}
Decisions regarding consumption over the lifespan require some estimate of how long that lifespan is likely to be. Payne et al. (2013) found that respondents' estimates of their own life expectancy are on average 8.6 years shorter when elicited using a die-by frame than when elicited by a live-to frame. If decision makers act on these life expectancies, then an arbitrary detail of framing will lead to drastically different choices. We propose that the framing effect is sensitive to the iterative probabilistic elicitation procedure employed by the previous literature. Study 1 compares the framing effect across the iterative probabilistic procedure and a point estimate procedure that simply asks respondents the age they will live to/ die by. The iterative probabilistic procedure implies a life expectancy six years shorter in the die-by frame than in the live-to frame, replicating the results of Payne et al. (2013). With the point estimate procedure, however, the framing effect reverses: the die-by frame increases life expectancy by three years. In Study 2 we test for the framing effect using a point estimate procedure on a representative sample of 2000 Britons. Again, and in contrast with the previous literature, we find that the dieby frame implies longer life. Our results reinforce the previous literature that beliefs around life expectancy are constructed. We recommend caution when attempting to debias life expectancy estimates or using life expectancies in choice architecture.
\end{abstract}

Keywords: Life Expectancy; Framing Effects; Probabilistic Reasoning; Annuities

\section{Word count: $\mathbf{6 , 7 5 8}$}


Judgments of life expectancy are implicit in many of life's largest decisions (e.g. retirement saving and decumulation decisions; insurance purchase; house purchase; health behaviors etc.). The dominant models of life-cycle consumption assume that agents maximize the stream of utility over a lifespan that is known to the consumer ex ante (e.g. Benartzi \& Thaler, 2007; Browning \& Crossley, 2001, Modigliani, 1986).

Recently, researchers have found that individuals' estimates of their own life expectancy are far less stable than had been assumed in life-cycle models. Across three studies of over 2000 people, elicitation using a die-by frame yielded an average implied life expectancy of 67 , whereas a live-to frame yielded a life expectancy of 76 (Payne et a.1, 2013). The scale of this framing effect suggests dramatic implications for decisions regarding retirement savings and decumulation. Given that the US Social Security Administration calculates the Normal Retirement Age for people born after 1960 to be 67 (Office of the Chief Actuary, n.d.), the average respondent in Payne et al.'s live-to frame implied that their retirement savings would have to last over nine times as long as the average respondent in the die-by frame. If Payne et al.'s result pervades decision making, there are likely to be many households whose life plans are premised on biased information. One indication that Payne et al's results generalize is that the framing effect has been replicated on samples of 2000 respondents in each of the Netherlands and Australia (Teppa, Thorpe \& Bateman, 2015).

\section{Generalizability}

The current research points to a methodological feature of previous research on the liveto/ die-by framing effect that has two important implications for its generalizability. Previous research elicits life expectancies using an iterative probabilistic procedure. Payne et al. (2013), Shu and Payne (2015) and Teppa et al. (2015) ask respondents to report the percentage chance 
that they would live to [die by] 65 or older [younger]; then repeat the process for ages 75,85 etc.

We are concerned that this probabilistic method is 1) likely to exacerbate framing effects; and 2) unlikely to represent the method that people spontaneously adopt when making important life decisions. The remainder of this paper presents two studies that investigate our first concern experimentally: we test whether the probabilistic method exacerbates framing effects relative to a point estimate approach to estimating life expectancy. For the next paragraph we consider whether people are likely to spontaneously estimate life expectancy using probabilities.

$\mathrm{Hu}$ and Odean (2011) point out that "anyone planning for retirement must answer an impossible question 'How long will I live?'”. At issue is whether people answer that question by an iterative probabilistic procedure. That they do not is suggested by a wealth of research documenting that people are cognitive misers: they adopt strategies, termed heuristics, that reduce the effort required in judgment and decision making (e.g. Fiske \& Taylor, 1991; Kahneman \& Frederick, 2002; Shah \& Oppenheimer, 2008; Simon, 1990). There are many papers documenting that people make complex judgments by spontaneously asking themselves easy-to-answer questions (for a review see Kahneman \& Frederick, 2002). For instance, when asked if they believe in climate change, lay people answer as if asked is today unusually hot? (Li, Johnson, \& Zaval, 2012). We have not encountered any evidence that people spontaneously ask themselves questions that require more steps and that are more difficult to calculate than other more intuitively phrased questions that get at the same underlying construct. Hu and Odean expressed the implicit question of life expectancy as how long will I live? Here in our study, we ask at what age will I live/ die? Each of these questions paraphrases the relevant question: what is my best estimate of my lifespan? to generate a point estimate in terms of years. The probabilistic question what is the percentage chance I will live to/ die by 65/75/85 etc.? does 
not paraphrase the relevant question, nor does it even yield answers on the same scale; translating probabilities into chronological years requires a mathematical transformation of parameter estimates returned from a regression. The reader will recognize that the iterative probabilistic procedure is a less intuitive, more effort-intensive and more computation-intensive approach to estimating life expectancy than the point estimate procedures suggested by $\mathrm{Hu}$ and Odean (2011) and by us. We consider it unlikely that people spontaneously estimate their age of death with reference to probabilities because the alternative point estimate procedure is a more obvious approach, it gets at the estimate in fewer steps, and it requires less computation. Of course, this does not preclude the possibility that the probabilistic procedure is more representative of spontaneous judgment processes used for certain tasks. For instance, when it comes to a question regarding a specific age or event (e.g. "will I be alive to see my grandson graduate?") people may spontaneously answer with some subjective likelihood estimate.

However, for the task at issue - estimating life expectancies - we suggest that a point estimate procedure is the more likely candidate.

Notwithstanding our concerns about how well the iterative probabilistic method captures spontaneous judgment processes, its use in previous studies is understandable because the influential Health and Retirement Study (henceforth HRS), a longitudinal survey, has collected data by the iterative probabilistic method since the early 1990s. This longitudinal data allows analysis of whether self-reported probability of living to age 75 predicts death. The answer is that it does, though probabilities do not sufficiently update to take account of health shocks (Smith, Taylor \& Sloan, 2001) and men tend to be more optimistic than women (Hurd, 2009). More generally, concerns have been raised that HRS responses display inconsistencies relative to normative probability estimates (e.g. Hurd, 2009; Viscusi \& Hakes, 2003). 


\section{Burdensome Probabilities}

Where the HRS elicits life expectancies by the probabilistic procedure, the Survey of Consumer Finances asks “about how long do you think you will live?". Wu, Stevens and Thorp test the consistency of life expectancy implied by the point estimate procedure against the iterative probabilistic procedure, holding constant the live-to framing. They find "very low consistency" across these two measures that should yield identical estimates (Wu et al., 2015, p.26).

The result that peoples' probability estimates imply a different life expectancy than do their point estimates might be related to findings that suggest people find probabilities particularly difficult to work with. Inferences that are based on probabilities tend to be less normative than those based on natural frequencies (e.g. Gigerenzer \& Hoffrage, 2005). Laypeople tend to put less weight on statistical information when it is presented in a probabilistic format than when it is presented as a natural frequency (e.g. Fagerlin, Wang \& Ubel, 2005). In summary, a high degree of numeracy is required to accurately interpret information expressed in probabilities (Reyna \& Brainerd 2007).

The difficulties with probabilities evidenced in the previous paragraph are likely to be exacerbated when people are asked to report information in terms of probabilities. When Kahan et al (2012) asked a representative sample of the US population to convert the natural frequency 20 out of 100 to a percentage, 28 percent failed to do so accurately. Fischhoff and Bruine de Bruin document that some respondents report a 50\% chance to indicate that they have "no idea" of the true probability (1999). A consequence is that survey questions that elicit responses in 
terms of percentage probabilities tend to manifest an overrepresentation at 50\%, which Fishhoff and Bruine de Bruin term a “50\% blip". The pervasiveness of 50 percent blips in the HRS' life expectancy questions led the survey administrators to add a follow-up question so as to determine what respondents actually meant by answering " $50 \%$ ". In 2006, fully 23 percent of those who responded to the life expectancy question reported their probability of living to 75 as $50 \%$. Just 37 percent of those went on to answer that they thought it equally likely that they would die before reaching the age of 75 as die after they turned 75 (Hurd, 2009). Unfortunately, there was no follow up question to determine whether responding " $50 \%$ " overstated or understated each respondent's optimism of living beyond age 75 . We therefore cannot infer whether a bias was induced by the 50 blip. We can however infer that a substantial number of percentages in response to the iterative probabilistic procedure for eliciting life expectancy were misreported.

\section{Suggested Mechanisms}

We suggest three mechanisms through which probabilistic questions might exacerbate the live-to/ die-by framing effect. First, reporting biases in probability estimates could lead to an event-happens/ event-does-not-happen framing effect. Second, probabilistic questions seem especially susceptible to biased sampling of information. Third, the burden imposed by reporting probabilities could make respondents more prone to satisficing.

Reporting a probability involves mapping a likelihood judgment onto a numerical scale. A bias in that mapping process would be expected to lead to a live-to/ die-by framing effect. Consider a respondent who reports probabilities that are 5 percentage points higher than her underlying likelihood judgment. Take the case where her likelihood judgment of being alive at age $x$ is 15 percent. She would report a probability of being alive at age $x$ as $20 \%(=15 \%+5 \%$ 
bias). If reporting the complementary probability of being dead by age $x$, she would imply a probability of being alive at age $x$ of just $10 \%$. This occurs because her likelihood judgment of $15 \%$ delivers a complementary $85 \%$ chance of being dead and the reporting bias will inflate this to a $90 \%$ probability, which implies a $10 \%$ probability of being alive. This reporting bias will lead to longer life expectancies by the live-to frame than the die-by frame.

In reality reporting biases are likely to be less clean than in the stylized example above, but our point is that any bias in the mapping of subjective probabilities onto a numerical scale would deliver a live-to/ die-by framing effect, or more generally an event-happens/ event-doesnot-happen framing effect. For a more realistic example, we refer again to the HRS data to see how the use of round numbers could induce an event-happens/ event-does-not-happen framing effect.

When asked the probability that an event occurs, a respondent should use whichever number between 0 and 100 best represents her likelihood judgment. If an event is perceived as 1 percent more likely to occur than another event, we would like to see that it is reported as being 1 percent more likely. In practice, respondents tend to report round numbers. The 2006 HRS question that asked the percentage likelihood of being alive at age 75 saw 43 percent of responses cluster at just three points, $0 \%, 50 \%$ and $100 \%$ (Hurd, 2009). It seems likely that at least some of the 15 percent of respondents who reported $100 \%$ chance of living to 75 recognized that there is some risk of their dying before that age. This pattern of response suggests that some respondents are using round numbers to indicate qualitative judgments (e.g. "it is very likely"; "it is unlikely"), just as some respondents use 50\% to mean "I have no idea". In order that rounding does not lead to an event-happens/ event-does-not-happen framing effect it would have to be the case that the rounding errors observed by a positive frame are mirrored by 
complementary rounding errors by a negative frame e.g. respondents should be just as likely to report $0 \%$ chance of being dead at 75 as $100 \%$ chance of being alive at 75 .

The second category of reasons why a probabilistic question could exacerbate framing effects relates to a mechanism Payne et al. hypothesise - that the live-to frame calls to mind different information than the die-by frame. Questions that make salient one side of a binary outcome (e.g. "what is the chance you will live to age 85?"; "what is the chance it will remain dry tomorrow?") evoke two categories of queries: those supporting realization of that specific outcome and those against realization of that specific outcome. Because these questions draw attention to one possible outcome at the expense of the other, they lead to biased retrieval of queries from one category at the expense of the other, as predicted by Query Theory (Johnson, Haubl \& Keinen, 2007). Consequently, questions that focus on one side of a binary outcome cause that outcome to appear more likely, as evidenced in Mandel (2005), Yamagishi (2002) and most relevantly in Study 3 of Payne et al. (2013). The categories of queries evoked by the openended question "what age will you live to?" are less predictable and are likely to vary from one respondent to another. They might include for example "what age does someone of my sex and class typically die at?"; "do I have a family history of long life?"; "how healthy am I relative to the average person?" Given the range of possible queries that could be evoked by the point estimate procedure, Query Theory does not offer a definitive prediction on how point estimates will respond to the live-to/ die-by frame. For this reason, we expect the live-to/ die-by frame to induce more bias into the information retrieval process by the probabilistic procedure than it induces into the point estimate procedure.

Finally, we predict that respondents who have answered by the probabilistic procedure will have fewer cognitive resources with which to correct for biased information retrieval than 
those who answered by the point estimate procedure. Given the prior literature documenting respondents' difficulties reporting percentages it seems likely that cognitive resources are especially sapped by the task of estimating percentages. Additionally, the iterative probabilistic procedure entails answering four probabilistic questions. The effort this entails might leave fewer cognitive resources available for generating new queries, weighting queries appropriately and recognizing whether existing queries are representative (i.e. not biased). We would therefore expect to see an exacerbation of framing effects when respondents are asked to report percentages relative to when a less burdensome procedure is used.

In summary, we have outlined three reasons why the probabilistic procedure seems more susceptible to live-to/ die-by framing effects relative to a point estimate procedure. First, biased use of percentages could lead to an event-happens/ event-does-not-happen framing effect.

Second, their question wordings seem to invite biased sampling of information. Third, the burden they impose could provoke satisficing. We therefore believe that there is sufficient theoretical basis to pursue the question of whether a point estimate procedure yields similar framing effects to the iterative probabilistic procedure studied in previous research.

\section{Study 1}

Our study follows a 2 (live to/ die by frame) *2 (probabilistic/ point estimation) design. Our dependent variable is estimated age of death (henceforth fail age), as inferred from responses. In this study we test for the first of our proposed mechanisms - that there is a systematic interaction between the live-to/ die-by frame and errors in reports of percentages.

\subsection{Participants}

A convenience sample of UK residents aged over $18(N=563)$ were anonymously 
recruited online via Facebook, workplace email and the University of Stirling Portal. Eleven were removed for non-consent and 45 for non-UK residence. Thirty respondents dropped out before encountering the questions that elicited the dependent variable, and a further thirty who started answering the dependent variable failed to complete the remainder of the survey. This leaves 447 complete responses. As shall be explained more fully in the results section, a further thirty respondents had to be excluded from the analyses because their probability estimates did not admit estimation of fail age. This leaves a final sample size of 417 (60\% female; aged 18-87, $\left.M_{\text {age }}=38\right) 444$.

\subsection{Materials}

Participants completed an online survey hosted by SurveyGizmo, which elicited in order of appearance: age, sex, occupational status, then the life expectancy measure. There then followed other questions including 3 items from the PANAS scale, which are discussed in section 5. A full list of verbatim questions is presented in the Appendix. Lastly we asked whether the respondent had solicited help with the survey.

Respondents were randomly assigned to indicate life expectancy by one of the four procedures set out in table 1:

\section{[Table 1]}

Participants allocated to the probabilistic questions answered on horizontal slider-scales. We counterbalanced whether these ran from 0 to 100 or from $100-0$ in order to control for scale presentation effects, replicating Payne et al's design (2013). One difference between our design and Payne et al's is that all of our respondents reported probabilities in the same order (i.e. age 65 , then $75,85, \&$ finally 95), whereas Payne et al varied the age for which respondents were 
first asked to report probability.

\subsection{Methods}

For respondents assigned to the point estimate procedure, the answer to the live to or die by question was taken as our dependent variable, fail age. For those assigned to the iterative probabilistic procedure, fail age was computed using the methodology reported in Payne et al (2013). We created a personal regression equation for each respondent from their reported probabilities of living to each of the ages in probabilistic questions $(65,75,85$ and 95) and, consistent with Payne et al, additional observations were added at the respondent's current age, for which a probability of 100 percent was imputed, and age 130 , for which probability of 0 percent was imputed. The parameter estimates from that regression were used to estimate a Weibull distribution curve, from which we could estimate the age at which the probability of dying is 50 percent, which was taken to be the fail age. A full explanation of the method used can be found in Appendix A of Payne et al 2013.

Our hypothesis is that fail age is predicted by a significant interaction between the iterative probabilistic procedure and the live-to/ die-by frame.

\subsection{Results}

Completion rates were similar across survey conditions. A logistic regression of drop out from the survey shows that neither the probabilistic iterative procedure nor the die-by frame has a significant effect (both $p \mathrm{~s}>.20$ ). Survey condition was not related to age or gender of respondents (all $p \mathrm{~s}>.20)$. 
The iterative probabilistic procedure elicited a large number of misreported percentages. Twenty-eight of the 220 respondents (12\%) using the probabilistic procedure reported higher probabilities of being alive at older ages than the probabilities they reported of being alive at younger ages. We were forced to drop these incoherent responses from the analysis because Weibull estimates could not be calculated for them. Consistent with Payne et al's methods, we retained the six respondents who implied equal probabilities of living to $65,75,85$ and 95 - in one case 100 percent probability, in three cases 50 percent probability, and 65 percent and 80 percent in the remaining cases.

In addition to the 28 respondents who gave incoherent probabilities, we were also forced to exclude from analyses two respondents for whom the Weibull distribution could not be calculated on account of their being aged over 65 . The first stage of the Weibull estimation procedure requires taking the log of the difference between 65 and current age. Because this difference is negative for respondents older than 65 , its $\log$ does not exist. In total, 30 respondents had to be dropped because Weibull distributions could not be calculated for them.

Figure 1 sets out the core result of study 1 . As can be observed, the live-to/ die-by framing effect was not merely attenuated by the point estimate procedure, but actually reversed. Figure 1 also graphs results from a follow-up Study 2, which replicated the reversal.

\section{[Figure 1: Life Expectancies by Survey Condition]}

Focussing first on the iterative probabilistic procedure, we replicated Payne et al's result: the die-by frame reduces life expectancy by 7 years relative to the live-to frame. Normal 
skewness and kurtosis was rejected for fail age and so in what follows we report the results of OLS regressions on a Box-Cox normalized dependent variable. Controlling for age, gender, occupation status and direction of probability scale (i.e. whether the response scale went from $100-0$ left-to-right), fail age by the die-by frame is significantly younger than by the live-to frame $\left[t=4.53, p<.001, \eta^{2}=.10\right]$. Direction of probability scale does not have an effect on estimated fail age $[t=0.73, p=.468]$, consistent with Payne et al's results.

By the point estimate procedure, the effect of the die-by frame reverses relative to the results reported above and in Payne et al (2013). The die-by frame now raises life expectancy by 3 years relative to the live-to frame. Controlling for age, gender and occupation status, fail age by the die-by frame is significantly older than by the live-to frame $\left[t=2.10, p=.037, \eta^{2}=.02\right]$.

Comparing fail ages across the live-to and die-by frames, it is clear that the hypothesized interaction between the probabilistic method and the live-to/ die-by frame is present. A regression in which we control for main effects of procedure and frame, age, gender, and occupation status confirms that the interaction of procedure and frame is statistically significant $\left[t=5.98, p<.001, \eta^{2}=.04\right]$

We now analyse whether the data support the mechanisms we proposed for why the probabilistic procedure would be especially prone to the live-to/ die-by framing effect. We suggested that working with percentages exerts a cognitive toll on respondents, which may inhibit correction for biased information retrieval. If there is in fact a greater cognitive burden on respondents in the probabilistic conditions, then we might expect that this burden would lead to fatigue and a higher rate of drop-out from the survey. Respondents in the probabilistic condition were in fact no more likely to drop out of the survey than those in the point estimate condition $[Z$ $=1.20, p=.23$ ). We would also expect that, if the probabilistic questions were particularly 
burdensome, they might make respondents more likely to seek help. More respondents (7.4\%) in the probabilistic condition reported having sought help than in the point estimate condition (4.1\%), though this difference is not statistically significant $[Z=1.38, p>.1]$. We cannot conclude, at least from these crude measures, that the probabilistic method was more cognitively taxing than the point estimate method.

We now test the second mechanism we proposed, that respondents' errors in reporting percentages are predicted by the framing manipulation. The first point to note on this is that there was a significant effect of the die-by frame on the coherence of reported probabilities. 24 of the 28 respondents who reported percentages that implied a higher probability of being alive at an older age than at a younger age answered by the die-by frame $[$ odds ratio $=4.53, Z(181)=2.32$, $p=.020]$.

Additionally, there is evidence to support rounding as a source of the event-happens/ event-does-not-happen framing effects. Of the 228 respondents aged under 65 who reported a probability of living to age 65, 21 indicated absolute certainty. Crucially, 18 of these absolute responses came from respondents in the live-to condition reporting $100 \%$ compared with just three from respondents in the die-by condition reporting $0 \%$. A logistic regression controlling for age, gender, occupation status and direction of probability scale shows this difference to be significant [odds ratio $=8.50, Z=3.21, p=.001]$. Generally, respondents answering by the liveto frame were more likely to use round numbers $(0 \%, 5 \%, 10 \%, 20 \%, 25 \%, 50 \%, 75 \%, 80 \%$, $90 \%, 95 \%$ or $100 \%$ ) than were those answering by the die-by frame [odds ratio $=2.21, \mathrm{Z}=2.62$, $\mathrm{p}=.009]$. These results suggest that reporting of probabilities interacts with the live-to/ die-by framing effect to generate systematic errors.

A similar test on reported point estimates of fail age shows no difference in the use of 
round numbers $(70,75,80,85,90,95,100)$ across the live-to and die-by frames $[Z=0.56, p=$ .573]. We return to consider what might drive the point estimate procedure to show higher life expectancies by the live-to frame than by the die-by frame in section 5 .

\section{Discussion}

Using the probabilistic procedure employed in previous studies, we replicate the framing effect found in those studies. The contribution of the current study is that it demonstrates that the live-to/ die-by framing effect is moderated by elicitation procedure.

We suggested three mechanisms through which probability estimates might exacerbate the live-to/ die-by framing effect, two of which were explored in our data analysis. First, we hypothesized that reporting probabilities would impose a burden on respondents. Our measures showed no evidence that respondents in the probabilistic condition were more burdened than those in the point-estimate condition. Second, we suggested that biased reporting of percentages could cause differences in estimated fail age across frames. We found respondents were more likely to report incoherent percentages by the die-by frame and were more likely to report rounded percentages (e.g. $80 \%$ rather than $82 \%$ ) by the live-to frame. In short, reporting errors do differ systematically across the live-to and die-by frames.

We did not explicitly test the third mechanism we proposed, which was that the question wording of the probabilistic procedure invites biased retrieval of information. That mechanism was tested, and support found for it, in Study 3 of Payne et al. (2013). For evidence that the point estimate procedure moderates this mechanism we point to the main results of the current study. Where the probabilistic procedure causes the live-to frame to evoke more queries in support of longer life than the die-by frame, figure 1 suggests that, if anything, the point estimate procedure causes the die-by frame to evoke more queries in support of longer life than the live-to frame. 
Though the scale of the framing effect by the point-estimate procedure was relatively small ( 3 years), it did attain statistical significance and so warrants a test for robustness. We report this in the next section.

\section{Study 2}

In study 2 we test for the live-to/ die-by effect using the point estimate procedure on a representative sample of UK residents.

\section{Participants}

A nationally representative sample of UK residents aged over $18(\mathrm{~N}=2096)$ undertook this four minute online survey as the first section in a multi-survey questionnaire, on 14-16 June 2016 ${ }^{1}$. The sample was selected from Populus's proprietary panel of 130,000 UK adults. Quotas were set on age, gender and region based on the known profile of Great Britain based on the 2012 National Readership Survey, a random probability face-to-face survey conducted annually with 34,000 adults. The characteristics of respondents in the live-to condition $\left(M_{\text {age }}=48,49 \%\right.$ female) did not differ significantly from those in the die-by condition $\left(M_{\text {age }}=49,50 \%\right.$ female, both $p \mathrm{~s}>.25)$.

\section{Methods}

As the first question in the survey, respondents were randomly allocated to one of four questions, of which two were the live-to/ die-by point estimate questions. The question wordings for these are precisely as they were in the previous study. The remaining conditions do not contribute to our research question ${ }^{2}$ and so for the current analysis we drop the respondents assigned to these conditions.

\footnotetext{
${ }^{1}$ This was shortly prior to the EU "Brexit" Referendum on $30^{\text {th }}$ June. Whilst there may have been some uncertainty resulting from the proximity to the ballot, we can think of no reason for this to have disproportionately affected any one branch of the treatment.

${ }^{2}$ They asked how long the respondent's pension would have to last and how many years of education the respondent had.
} 
Results

The pattern of results replicates that of Study 1 . The die-by fail age is again older $\left(M_{\text {die-by }}\right.$ $=83.5)$ than the live-to fail age $\left(M_{\text {live-to }}=81.7\right)$. Normal skewness and kurtosis was rejected for fail age and so we ran a bivariate OLS regression on a Box-Cox normalized dependent variable, which finds this difference to be statistically significant: $t=2.67, p=.008$. The survey provider furnished us with a large range of demographic measures: age, gender, education level, employment status, marital status, income category, disability status, religion and region. Controlling for these in a regression of the Box-Cox corrected variable, the die-by frame remains significant $\left[t=2.57, p=.010, \eta^{2}=.006\right]$.

\section{Some Results Regarding the Reversal}

We began this research with the hypothesis that requiring respondents to report probabilities would make them particularly susceptible to live-to/ die-by framing effects. We did not anticipate a reversal of the effect using the point estimate procedure. In study 2 , we prioritized testing the robustness of this reversal rather than its underlying mechanism with the result that we collected less information than we would have liked but from a larger and more representative sample. The reader might have correctly inferred from the header to this subsection that we cannot explain the specific mechanism by which the die-by frame elicits higher point estimates of fail age than the live-to frame. In this section we report some results that suggest a heuristic is at play and that might prove useful for future research on this question.

Table 2 summarizes fail ages from Studies 1 and 2 at various points in the distribution.

We had hoped to see some pattern common to both datasets, but whereas in Study 1 the effect of the die-by frame increases towards the top of the distribution, in Study 2 it remains fairly constant throughout. 


\section{[Table 2]}

We conducted exploratory tests using variables common to both datasets - age, gender and occupation status. None were found to moderate the effect. In study 1, we collected some affect items at the close of the survey: items on distress, afraid and enthusiasm from the PANAS scale (Watson, Clark and Tellegen, 1988). These affect measures do show significant moderation. Before reporting those moderation results, it is instructive to see how these affect items relate to our core variables. Consistent with the idea that dying is aversive, respondents who estimated lower fail ages also rated themselves higher on the distress and afraid scales and lower on the enthusiasm scale [OLS: distress: $t=3.33, p=.002$; afraid: $t=3.40, p=.001$; enthusiastic: $t=$ $3.12, p=.002]$. The live-to/ die-by manipulation did not cause a change in any of these affect measures (all $p \mathrm{~s}>.20)$.

Separate OLS regressions show that, after controlling for main effects, those who reported lower levels of distress and afraid demonstrated the largest response to the die-by-frame [distress interaction: $t=1.93, p=.055$; afraid interaction: $t=2.00, p=.047$ ] and those who reported higher levels of enthusiasm demonstrated larger effects in response to the die-by frame $[t=3.38, p=.001]$. The enthusiasm interaction emerges as significant from a model including all three affect measures and their interactions $[t=2.89, p=.004]$.

These results are consistent with research showing that positive affect results in less analytic processing, and hence greater reliance on heuristics, relative to negative affect (e.g. Ruder and Bless, 2003; Sinclair and Mark, 1995). One heuristic that has been helpfully suggested by an anonymous reviewer is based on Yaniv and Schul's observation that a stricter decision criterion is applied when judging what to include than when judging what to exclude (1997, 2000). Yaniv and Schul's model and experiments predict that there is a range of ages that 
would not meet the criterion to be included as plausible ages at which to still be alive but they will also not meet the criterion to be excluded as plausible ages at which to still be alive. In other words, these ages are included by the exclusionary approach, but not included by the inclusionary approach. If the die-by frame evokes an exclusionary approach and the live-to frame evokes an inclusionary approach then there is some range of ages at the top of the distribution that informs estimation of the age the respondent will die by, but does not inform estimation of the age the respondent will live to. What approach respondents actually take to answering the live-to/ die-by questions, and more generally to spontaneously estimating age of death, will have to wait for future research.

\section{General Discussion}

Our results provide further evidence of constructed beliefs in life expectancy. Specifically, they demonstrate that life expectancy estimates are sensitive not merely to whether they are elicited in a live-to or die-by frame, but are also sensitive to whether they are elicited probabilistically or as point estimates.

Whereas errors in reports of percentages and Query Theory offer an explanation for the direction of the framing effect in probabilistic questions, we can only speculate on why the liveto/ die-by framing effect reverses when elicited by point estimates.

We conclude by considering some real world implications of our results. Payne et al. (2013) express concern that the "10 year difference in the median expected age of being dead or alive is ... highly meaningful to a number of important life decisions, such as how to finance one's consumption during retirement". Our results offer some reassurance as well as some additional concern on this point. Further to Payne et al.'s concerns that estimates of life 
expectancy are not stable, we add that the framing effect itself is not stable. A striking result is apparent from focussing on participants in the die-by conditions of our Study 1: answering the probabilistic-iterative procedure relative to the point-estimate procedure reduces life expectancy by nine years (see figure 1).

Our first implication concerns analyses of secondary data on life expectancy. The discrepancy across the probabilistic-iterative procedure and the point estimate procedure has implications for the validity of research that uses subjective life expectancy as a variable. The Health and Retirement Survey uses the probabilistic-iterative procedure and the Survey of Consumer Finances uses the point estimate procedure. A search on Google Scholar ${ }^{3}$ for the exact phrases "Health and Retirement Survey" and "life expectancy" returned 1910 results. A search for "Survey of Consumer Finances" and "life expectancy" returned 1780. Our results suggest that these analyses will have been sensitive to the procedure that was used to elicit life expectancy, and that the distortions induced by these procedures may have been large.

Our second implication is for measurement of subjective estimates of life expectancy. One advantage of the iterative probabilistic procedure is that it delivers a probability distribution of fail ages for each respondent. Its disadvantages are substantial however. First, the resultant probability distributions are of questionable validity since they are biased by information retrieval and in many cases they are derived from misreported percentages. Second, the procedure is costly in terms of missing data: in Study 1 we lost estimated fail ages for 14 percent of the probabilistic conditions' sample because Weibull distributions could not be calculated for 30 out of 220 observations. We recommend development of a new means to elicit distributions of life expectancies. On the basis of the current results, we would recommend that this procedure does not ask respondents to report percentages.

\footnotetext{
${ }^{3}$ Conducted on October $19^{\text {th }} 2016$
} 
A third implication is a note of caution on applying the live-to/ die-by frame as a piece of choice architecture to nudge decision making. In some circumstances die-by frames increase life expectancy and in others they decrease life expectancy. This reversal should give policymakers pause before attempting to apply these framing effects as nudges.

We finish with a question for future research. We found that the framing effect manifested by point estimate procedures is smaller in scale than that returned by the iterative probabilistic procedure. If our logic is correct that people are unlikely to spontaneously employ the iterative probabilistic procedure to estimate life expectancy when making decisions, then the difference in expected age of being dead or alive is likely to be substantially less than that suggested by the previous literature, and to that extent, less of a source of bias in decision making. However, it is possible that people spontaneously employ a live-to frame in some circumstances (e.g. when deciding whether to buy a vacation home) and a die-by frame in others (e.g. when deciding to annuitize or take a lump-sum payment). An important question for future research is to determine how spontaneous judgments of life expectancy are formed.

\section{References:}

Benartzi, S., \& Thaler, R. H. (2007). Heuristics and biases in retirement savings behavior. The Journal of Economic Perspectives, 21(3), 81-104.

Browning, M., \& Crossley, T. F. (2001). The life-cycle model of consumption and saving.The Journal of Economic Perspectives, 15(3), 3-22.

Fagerlin, A., Wang, C., \& Ubel, P. A. (2005). Reducing the influence of anecdotal reasoning on people's health care decisions: is a picture worth a thousand statistics? Medical Decision Making, 25(4), 398-405.

Fischhoff, B., \& De Bruin, W. B. (1999). Fifty-fifty= 50\%? Journal of Behavioral Decision Making,12(2), 149-163. 
Fiske, S. T., \& Taylor, S. E. (2013). Social cognition: From brains to culture. NYC: NY Sage.

Gigerenzer, G., \& Hoffrage, U. (1995). How to improve Bayesian reasoning without instruction: Frequency formats. Psychological Review, 102(4), 684.

Hu, H.T., \& Odean, T. (2011). Paying for old age. New York Times 2/25/2011.

Hurd, M. D. (2009). Subjective probabilities in household surveys. Annual Review of Economics, 1, 543.

Kahneman, D., \& Frederick, S. (2002). Representativeness revisited: Attribute substitution in intuitive judgment. In T. Gilovich, D. Griffin, \& D. Kahneman (Eds.), Heuristic and biases: The psychology of intuitive judgment (pp. 49-81). New York: Cambridge University Press.

Johnson, E. J., Häubl, G., \& Keinan, A. (2007). Aspects of endowment: a query theory of value construction. Journal of Experimental Psychology: Learning, Memory, and Cognition, 33(3), 461-474.

Li, Y., Johnson, E.J., \& Zaval, L., (2011). Local warming daily temperature change influences belief in global warming. Psychological Science, 22, 454-459.

Mandel, D. R. (2005). Are risk assessments of a terrorist attack coherent?. Journal of Experimental Psychology: Applied, 11(4), 277.

Modigliani, F. (1986). Life cycle, individual thrift, and the wealth of nations. The American Economic Review, 76(3), 297-313.

Office of the Chief Actuary (n.d.) accessed 7/21/16 https://web.archive.org/web/20160721075851/https://www.ssa.gov/OACT/ProgData/nra.html

Payne, J. W., Sagara, N., Shu, S. B., Appelt, K. C., \& Johnson, E. J. (2013). Life expectancy as a constructed belief: Evidence of a live-to or die-by framing effect. Journal of Risk and Uncertainty, 46(1), 27-50.

Peters, E., Västfjäll, D., Slovic, P., Mertz, C. K., Mazzocco, K., \& Dickert, S. (2006). Numeracy and decision making. Psychological Science, 17(5), 407-413.

Reyna, V. F., \& Brainerd, C. J. (2007). The importance of mathematics in health and human judgment: Numeracy, risk communication, and medical decision making. Learning and Individual Differences, 17(2), 147-159.

Ruder, M., \& Bless, H. (2003). Mood and the reliance on the ease of retrieval heuristic. Journal of Personality and Social Psychology, 85(1), 20-32. 
Shah, A. K., \& Oppenheimer, D. M. (2008). Heuristics made easy: an effort-reduction framework. Psychological Bulletin, 134(2), 207.

Shu, S. \& Payne, J (2015). Psychological Factors in Savings and Decumulation Decisions, in Kristin Diehl and Carolyn Yoon (Ed.s) NA - Advances in Consumer Research, 43, (Pp: 136-141). Duluth, MN: Association for Consumer Research.

Sinclair, R. C., \& Mark, M. M. (1995). The effects of mood state on judgemental accuracy: Processing strategy as a mechanism. Cognition \& Emotion, 9(5), 417-438.

Simon, H. (1990). Reason in human affairs. Stanford University Press.

Smith, V. K., Taylor, D. H., \& Sloan, F. A. (2001). Longevity expectations and death: Can people predict their own demise?. The American Economic Review, 91(4), 1126-1134.

Teppa, F., Thorp, S., \& Bateman, H. (2015). Family, Friends and Framing: A Cross-Country Study of Subjective Survival Expectations. UNSW Business School Research Paper, (2015ACTL19).

Viscusi, W. K., \& Hakes, J. (2003). Risk ratings that do not measure probabilities. Journal of Risk Research, 6(1), 23-43.

Wu, S., Stevens, R., \& Thorp, S. (2015). Cohort and target age effects on subjective survival probabilities: Implications for models of the retirement phase. Journal of Economic Dynamics and Control, 55, 39-56.

Yamagishi, K. (2002). Proximity, compatibility, and noncomplementarity in subjective probability. Organizational Behavior and Human Decision Processes, 87(1), 136-155.

Yaniv, I., \& Schul, Y. (1997). Elimination and inclusion procedures in judgment. Journal of Behavioral Decision Making, 10(3), 211-220.

Yaniv, I., \& Schul, Y. (2000). Acceptance and elimination procedures in choice:

Noncomplementarity and the role of implied status quo. Organizational Behavior and Human Decision Processes, 82(2), 293-313. 


\begin{tabular}{|c|c|}
\hline \multicolumn{2}{|c|}{ Table 1} \\
\hline Procedure & Wording \\
\hline $\begin{array}{l}\text { Probabilistic } \\
\text { live-to }\end{array}$ & "The chance that I will live to be $65 / 75 / 85 / 95$ years old or older is \\
\hline $\begin{array}{l}\text { Probabilistic } \\
\text { die-by }\end{array}$ & "The chance that I will die at $65 / 75 / 85 / 95$ years old or younger is \\
\hline Point live-to & "I expect to live to age \\
\hline Point die-by & "I expect to die by age \\
\hline
\end{tabular}


1

2

3

4

5

6

7

8

9

10

11

12

13

14

15

16

17

18

19

20

21

22

23

24

25

26

27

28

29

30

31

32

33

34

35

36

37

38

39

40

41

42

43

44

45

46

47

48

49

50

51

52

53

54

55

56

57

58

59

60

\begin{tabular}{|c|c|c|c|c|}
\hline \multicolumn{5}{|c|}{$\begin{array}{l}\text { Table } 2 \\
\text { Point estimates of life expectancy at various points in the distribution }\end{array}$} \\
\hline & \multicolumn{2}{|c|}{ Study 1} & \multicolumn{2}{|c|}{ Study 2} \\
\hline & Live-to & Die-by & Live-to & Die-by \\
\hline $25^{\text {th }}$ percentile & 78.5 & 78 & 75 & 79 \\
\hline $50^{\text {th }}$ percentile & 84 & 84.5 & 80 & 85 \\
\hline $75^{\text {th }}$ percentile & 88 & 90 & 88 & 90 \\
\hline $90^{\text {th }}$ percentile & 90 & 97 & 95 & 98 \\
\hline
\end{tabular}


Figure 1: Life Expectancies by Survey Condition

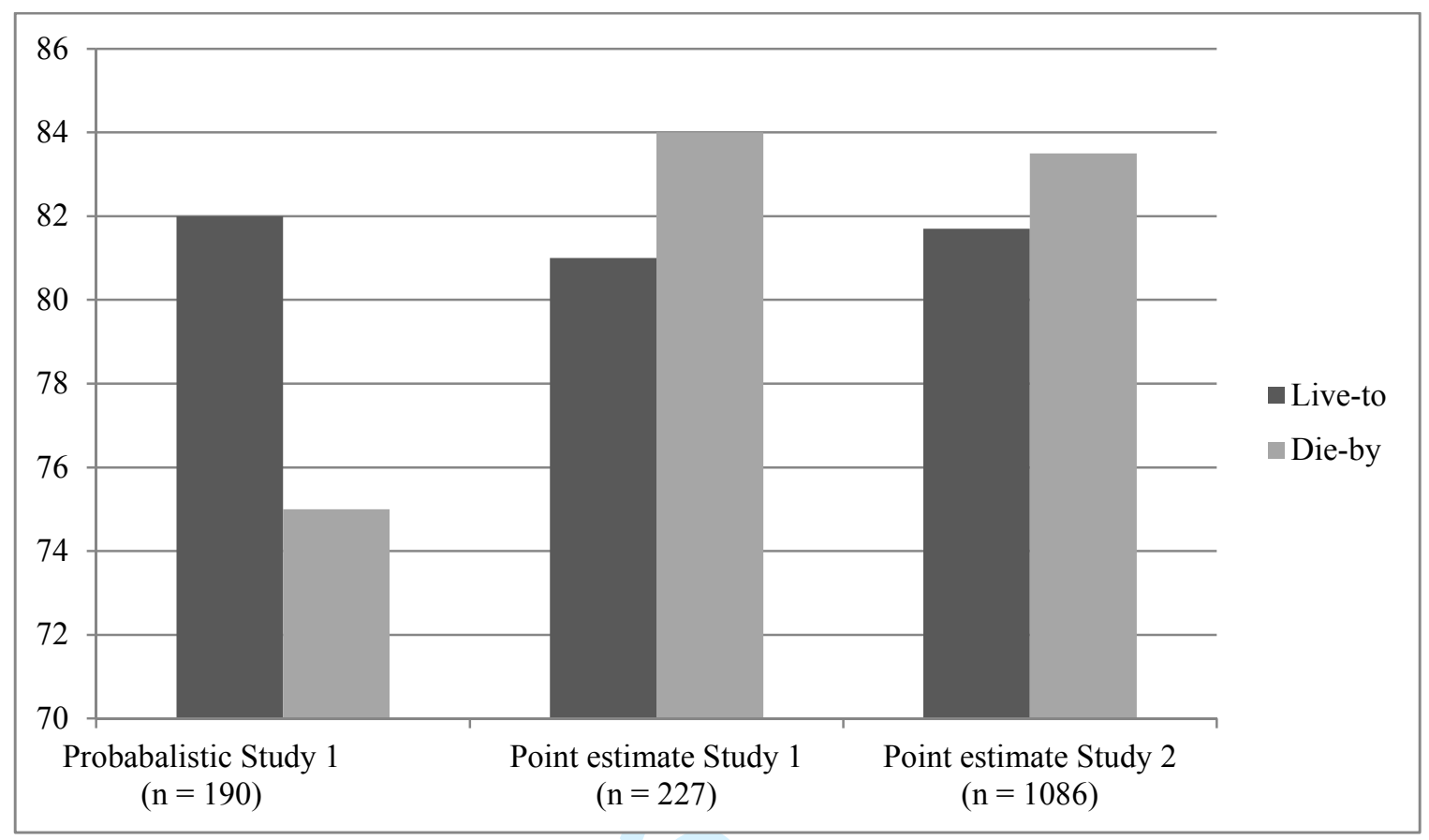

Fig. 1: Mean Estimated Fail Ages (life expectancies) from the Probabilistic Iterative Procedure (far left) and Point Estimate procedures (center and right). Differences in sample sizes across conditions in Study 1 are accounted for by 30 respondents in the probabilistic conditions for whom it was impossible to estimate fail ages. 